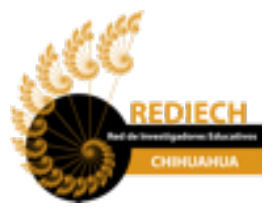

Red de Investigadores Educativos Chihuahua A.C. Chihuahua, México www.rediech.org

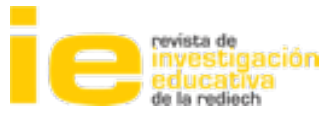

ISSN: 2007-4336

ISSN-e: $2448-8550$

http://www.rediech.org/ojs/2017/index.php/ie rie rediech/index

Felipe de Jesús Jasso Peña

José Porfirio Tamez Solís

José Antonio Torres Reyes

2019

\title{
Metahabilidades en información y evolución conceptual en la educación virtual
}

IE Revista de Investigación Educativa de la REDIECH, 10(18), pp. 23-42.

http://dx.doi.org/10.33010/ie_rie_rediech.v10i18.165

\section{(c) $(1) \Theta$}

Esta obra está bajo licencia internacional

Creative Commons Reconocimiento-NoComercial 4.0. 


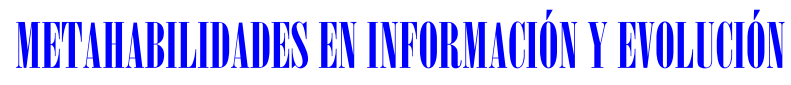

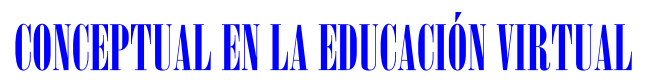

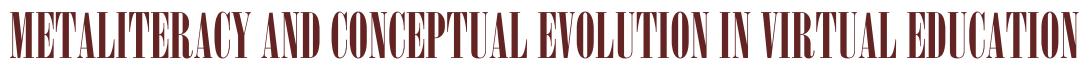

\author{
JASSO PEÑA Felipe de Jesús \\ TAMEZ SOLÍS José Porfirio \\ TORRES REYES José Antonio
}

Recepción: junio 4 de 2018 | Aprobado para publicación: Septiembre 29 de 2018

\section{Resumen}

Aunque internacionalmente se ha destacado la importancia que tiene la habilidad para el acceso, uso y distribución de la información en el aprendizaje de nuevos conceptos en un ambiente universitario hace falta fomentar la integración de su instrucción en el currículo e investigar sus alcances didácticos. Por ello, en este estudio de corte mixto-exploratorio se buscó comprender la manera en que el desarrollo de las metahabilidades en información (MTHI) coadyuvan con la evolución conceptual en estudiantes de posgrado virtual, encontrándose elementos instruccionales que muestran cierta relación entre el desarrollo de las MTHI con el desarrollo de conocimientos, además del valor de la participación del profesor bibliotecario como un cotutor que apoye a los

Felipe de Jesús Jaso Peña. Coordinador de formación y atención a usuarios de los programas de Posgrado de Educación Digital del Instituto Tecnológico y de Estudios Superiores de Monterrey, Nuevo León, México. Tiene estudios de maestría en educación, bibliotecología y ciencias de la información, enseñanza superior y en administración pública. Las principales actividades laborales están relacionadas con la docencia y el trabajo bibliotecario. Correo electrónico: fjasso@itesm.mx. ID: http://orcid.org/0000-0002-8148-8399.

José Porfirio Tamez Solís. Profesor asociado de tiempo completo en la Universidad Autónoma de Nuevo León, México. Desarrolla actividades de docencia, investigación, tutoría y gestión académica y administrativa. Director de tesis de alumnos de maestría y doctorado. Ha prestado asesorías sobre diseño y operación de sistemas bibliotecarios, programas de formación de personal bibliotecario y diseño y construcción de edificios para bibliotecas. Colaborador de organizaciones académicas como Orión (Argentina), ALIAT Universidades (México) y el Centro de Estudios Legislativos del Congreso del Estado de Nuevo León. Correo electrónico: porfirio.tamezs@uanl.mx. ID: http://orcid. org/0000-0003-4848-1854..

José Antonio Torres Reyes. Profesor-investigador de tiempo completo en la Facultad de Filosofía y Letras de la Universidad Autónoma de Nuevo León, México. Es doctor en información científica por la Universidad de Granada, España. Ha publicado artículos en revistas especializadas, memorias de conferencias y capítulos de libros. Es evaluador de la Asociación para la Acreditación y Certificación en Ciencias Sociales AC (Acceciso). Fue miembro del Copy South Research Group de la University of Kent at Canterbury, Reino Unido. Es editor de publicaciones literarias y de cultura general. Correo electrónico: jose.torresrys@uanl.edu.mx. ID: http://orcid.org/0000-0002-0545-7865. 
alumnos en la adopción y perfeccionamiento de un proceso reflexivo de rastreo informativo.

\author{
Palabras clave: HABILIDADES EN INFORMACIÓN, FORMACIÓN DE \\ CONCEPTOS, PROFESOR BIBLIOTECARIO.
}

\begin{abstract}
Although the importance of the ability to access, use and distribution of the information on learning of new concepts in a university environment has been emphasized internationally, it is necessary to encourage the integration of its instruction into the curriculum and research their educational scopes. Therefore, in this mixed-exploratory study, we sought to understand the way in which the development of metaliteracy skills contribute to the conceptual evolution in students of virtual postgraduate, finding instructional elements that show a certain relationship between the development of the metaliteracy skills with the development of knowledge, in addition to the value of the participation of the teacher librarian as a tutor assistant that supports the students in the acquisition and improvement of a reflective process of informative tracking.
\end{abstract}

Keywords: INFORMATION LITERACY, CONCEPT FORMATION, TEACHER
LIBRARIAN.

\title{
IITroulcociós
}

Dentro de los principales objetivos de la educación superior se encuentran la formación de científicos profesionales, la alfabetización científica de la población y la transmisión del acervo del conocimiento científico acumulado a lo largo de la historia (Fraser, 2016; Taber y Akpan, 2017). Por este motivo, se espera que los estudiantes universitarios desarrollen (con apoyo de sus profesores) las habilidades necesarias para ingresar al pensamiento científico a través de ejercicios de investigación, revisión de bibliografía especializada e impulsando su participación en eventos de corte científico como conferencias, simposios y coloquios. Sin embargo, de acuerdo con Delaney y Bates (2017), dada la gran cantidad de actividades y responsabilidades que tiene el docente, regularmente su orientación se limita a la bibliografía que conoce, acorde con su experiencia docente e investigador, y alienta al alumnado a que realice búsquedas por su cuenta como, por ejemplo, la revisión del estado del arte de algún tema en particular. Las dificultades surgen cuando se carece de instrucción acerca de cómo localizar ese material, considerando que el profesor (regularmente) no incluye dentro de los contenidos de curso estrategias acerca del rastreo de información en las herramientas y fuentes disponibles. Por ello hacen falta propuestas para educar en la recuperación de información (Richardson et al., 2015).

Precisamente, ante esta necesidad de instrucción ha surgido la figura del pro24 fesor-bibliotecario (teacher librarian), profesionista con estudios en educación y 
en bibliotecología, responsable de capacitar a los estudiantes (y a los mismos docentes) para ser autosuficientes en el rastreo informativo (Godfree y Neilson, 2018; McAdoo, 2010). Este apoyo se ha tecnificado y profesionalizado de tal manera que ya no se limita a la educación presencial, sino que se ha extendido a la virtualidad, aprovechando las tecnologías de información y comunicación modernas, buscando estrechar la colaboración entre la academia y la biblioteca. Lo anterior con la finalidad de desarrollar actividades académicas en conjunto que apoyen la investigación a través de una exploración adecuada de las fuentes de información más relevantes (Ángel, 2016). Es aquí, precisamente, en donde se explora la utilidad del desarrollo de las metahabilidades en información (MTHI) como una estrategia para la educación científica en estudiantes de orden superior en su primer año de ingreso, durante su carrera y el posgrado (Fulkerson, Ariew y Jacobson, 2017; Majetic y Pellegrino, 2014).

Para concretar lo señalado, el estudio tuvo como objetivo demostrar que, a través del desarrollo de las MTHI en alumnos de posgrado virtual, es posible coadyuvar con su autodirección en el descubrimiento, comprensión, aprendizaje y evolución conceptual (Jacobson y Mackey, 2016). De hecho, el fomento de la autodirección en la educación no es un tema nuevo. Hace más de dos mil años Platón describe (en La apología de Sócrates) cómo a este último se le acusaba de animar a los jóvenes a responsabilizarse de su propio pensamiento con tal de volverse reflexivos y críticos de las prácticas tradicionales, además de esforzarse por llegar a determinar conceptos universales, tales como: la virtud, la valentía, la piedad o el deber (Álvarez, 2016; Jeager, 2015).

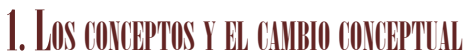

Un concepto es un constructo mental o representación de categorías y se define como una clase de objetos o de hechos que comparten una o más similitudes, también llamadas atributos (Corsini, 2016). Se pueden distinguir dos tipos de conceptos; estos son (Aparicio y Rodríguez, 2015):

a) Concretos o naturales, que se conforman mediante la experiencia con relación al objeto o evento que se trate. Por ello, una vez que son asimiladas las características que los distinguen, pueden ser identificados en cualquier otro momento.

b) Los abstractos; requieren un procesamiento más complejo, mismo que una percepción clara de las características concretas no permite una adecuada identificación. Esta es la razón por la cual estos conceptos requieren de la adquisición de una definición formal (Fodor y Pylyshyn, 2015; Leliwa, Scangarello y Ferreyra, 2016).

Los conceptos también son considerados como bloques que colaboran en la construcción del pensamiento, ya que ayudan en la elaboración de representaciones de objetos, eventos y actividades (Margolis, 2016). Asimismo, facilitan una organización sistemática de la información en categorías y además previenen el reaprendizaje; esto significa que, por ejemplo, una vez apropiándose del concepto de "perro", no es necesario volver a aprehenderlo al encontrarse con uno de ellos (Devine, 2016).

Por su parte, el término cambio conceptual es una de las expresiones utilizadas con mayor frecuencia en la enseñanza de la ciencia. De acuerdo con Posner et al. 
(1982), investigadores que forman parte de los iniciadores de la propuesta de enseñanza del cambio conceptual, uno de los puntos importantes a considerar radica en cómo cambian las concepciones al recibir el impacto de los nuevos conceptos y evidencias. Aunque años después Strike y Posner (1992) hicieron una revisión a su propuesta original, apreciando ahora al cambio conceptual como el resultado de una evolución y no meramente de una eliminación de los conceptos anteriores por unos nuevos. En ese mismo sentido, esta propuesta didáctica ha sido analizada desde varios puntos vista, mismos que se dividen en dos tipos (Marín, 1999): a) las críticas que se realizan en el plano de la enseñanza, evaluando resultados, apreciando incoherencias 0 restricciones y resaltando las dificultades para alcanzar objetivos de enseñanza; $y$, b) señalamientos sobre las bases epistemológicas que le dan fundamento.

Una postura interesante, y que tiene elementos muy útiles para el desarrollo de conocimientos, es el aprendizaje por descubrimiento propuesta por Bruner mediante la autorregulación del comportamiento (Cattaneo, 2017). Por otro lado, Moreira y Greca (2003) hacen un análisis del desarrollo teórico del cambio conceptual y se alejan de las propuestas del conflicto cognitivo de Piaget (2015) y de las concepciones existentes insatisfechas de Posner et al. (1982) para apoyar el modelo de Thomas Kuhn (2006) y buscar una transformación del constructo cambio conceptual a evolución conceptual a través de la teoría del aprendizaje significativo de Ausubel, Novak y Hanesian (1990). En ese mismo sentido, Pozo (2006 y 2008) hace énfasis en que el cambio conceptual requiere tener acceso a nuevos formatos y sistemas de representación diferentes a los que forman la estructura de nuestras teorías primigenias (Webb y Hayhoe, 2018).

Sea cual fuera la postura teórica, ya sea del cambio o evolución conceptual, este proceso de asimilación y restructuración de nuevos conceptos ofrece (tanto al estudiante como a sus profesores) un reto para el desarrollo de conocimiento nuevo, sobre todo a través de la investigación científica, que no siempre es bien recibida y percibida por los alumnos universitarios (Bellová, Melicherčíková y Tomčík, 2017). Ante estos requerimientos de organización y adaptación de los conocimientos de los estudiantes regularmente se presenta lo que Perkins (2008, p. 37) denomina sindrome del conocimiento frágil, del cual describe sus características:

- Conocimiento olvidado: en ocasiones, buena parte del conocimiento simplemente se esfuma.

- Conocimiento inerte: a veces se le recuerda, pero es inerte; permite a los estudiantes aprobar los exámenes, pero no se lo aplica en otras situaciones.

- Conocimiento ingenuo: el conocimiento suele tomar la forma de teorías ingenuas o estereotipos, incluso luego de haber recibido una instrucción considerable, destinada especialmente a proporcionar mejores teorías y a combatir los estereotipos.

- Conocimiento ritual: los conocimientos que los alumnos adquieren tienen con frecuencia un carácter ritual que solo sirve para cumplir con las tareas escolares. Así, una forma de analizar el proceso de elaboración conceptual radica en la alfabetización científica, cuyas estrategias buscan evitar la memorización de datos y definiciones. En su lugar, se prefiere el desarrollo de un conocimiento del método 26 y conceptos científicos (Hicks, MacDonald y Martin, 2017; Ozdem-Yilmaz y Cavas, 
Fig. 1. El sistema cognitivo.

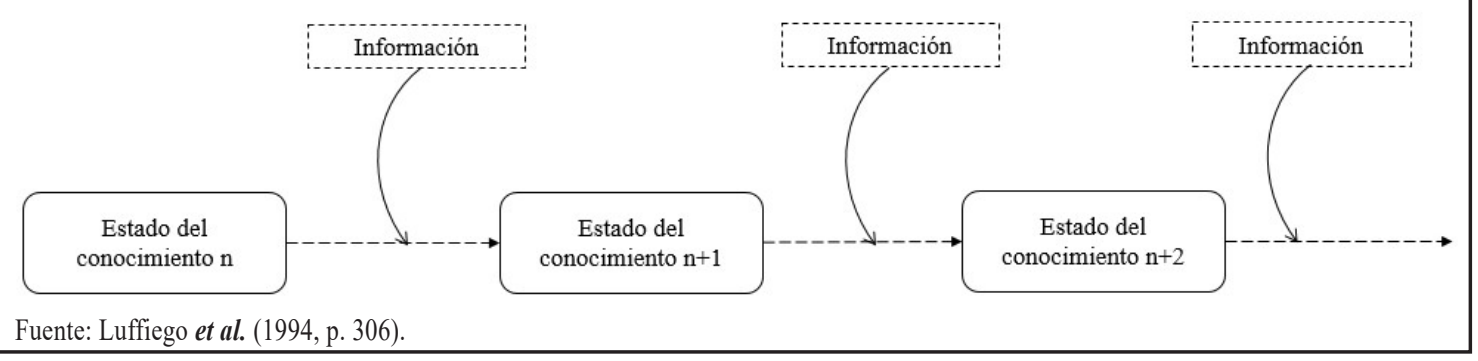

2016). No obstante, Webb y Hayhoe (2018) mencionan que los alumnos traen consigo ideas preconcebidas acerca del funcionamiento del mundo $\mathrm{y}$, como consecuencia, se les dificulta la comprensión de los nuevos conceptos, obstaculizando su aprendizaje. De ahí la importancia de un planteamiento metacognitivo de instrucción que les apoye a controlar su propio aprendizaje, definir sus objetivos y supervisar sus avances (Ferreira, El-Hani y Silva-Filho, 2016; Mayor, Suengas y González, 1993).

Es verdad que existe una amplia variedad de investigaciones para explicar el cambio o modificación conceptual, por lo que una forma de organizarlas para observar sus características generales la proporcionan Özdemir y Clark (2007), al dividirlas en dos grandes grupos: a) propuestas centradas en el conflicto cognitivo; $\mathrm{y}, \mathrm{b}$ ) elementos fenomenológicos, factores, facetas, narrativas, modelos mentales y etapas del desarrollo. Este último grupo es considerado por el "modelo sistemático de evolución conceptual" desarrollado por Luffiego et al. (1994). Para estos autores, el conocimiento pasa de una relativa estabilidad a una inestabilidad que, poco a poco, busca regresar a un equilibrio, no sin antes verse influido (adquisición y pérdida de conocimientos) por diversas variables, tales como: la motivación, la formación, la estructura conceptual, la inteligencia, el tipo de educación y la condición social (ver figura 1).

Por lo tanto, la evolución conceptual es el proceso mediante el cual se incorpora nueva información al sistema cognitivo mediante la presencia de un captador conceptual (concept attractors) relevante que provoque un desequilibrio (Oliva, 1999), por lo que el sistema cognitivo buscará reorganizar dicho captador mediante pequeños ajustes sin modificar su núcleo. Sin embargo, si acaso no es posible hacerlo, entonces se procede a reestructurarlo con nueva información que permita regresar a un equilibrio. Aquí es donde interviene la diferencia que existe entre cada individuo para el desarrollo de nuevos conceptos, ya que como Luffiego et al. (1994) afirman, dicho aprendizaje es impredecible debido a que cada sujeto aprecia de manera distinta el fenómeno de donde provienen dichos conceptos y su capacidad para esquematizarlos.

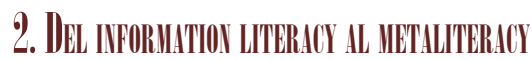

El concepto de las habilidades en información (information literacy) fue propuesto por primera vez por Paul Zurkowsky $(1974$, p. 6) en el marco de un congreso empresarial, en donde hace el siguiente señalamiento: "[...] people trained in the 
application of the information resources to their work can be called information literates" (las personas formadas en la aplicación de los recursos de información para su trabajo pueden llamarse alfabetizados en información). De hecho, actualmente este tipo de competencias son requeridas en la actividad laboral actual (Gilbert, 2017) y forman parte de los requisitos para llegar a ser un ciudadano global (Goodiera, Field y Goodman, 2018).

Por su parte, Bruce (2017) y Brodie (2017) acentúan la importancia de la habilidad relacionada con la recuperación de la información debido a que, sin ella, no podría haber ningún tipo de avance en la formación científica. La descripción de este tipo de habilidades dio como resultado el estudio del comportamiento informativo (information behavior) y su influencia sobre la forma en que la persona resuelve sus requerimientos de datos. Este concepto se refiere al comportamiento activo o pasivo del individuo en cuanto a la búsqueda y recuperación de información. Esto incluye las conversaciones con otras personas o fuentes como la televisión, publicidad y toda aquella información que no se busca con alguna intención específica (Ford, 2015).

Actualmente existen diversos organismos internacionales que proponen estándares para el desarrollo de las habilidades en información considerando su transversalidad en la educación (ACRL, 2017); entre ellas se encuentran: la American Library Association y la Association of College y Research Libraries; la Society of College, National and University Libraries (SCONUL) Working Group on Information Literacy en el Reino Unido; la Australian and New Zealand Institute for Information Literacy en conjunto con el Council of Australian University Librarians; la Federación Internacional de Asociaciones e Instituciones Bibliotecarias y los 7 objetivos de las metahabilidades en información (metaliteracy) propuestas por Mackey y Jacobson (2014).

Todas estas propuestas surgieron debido a la necesidad de aprovechar las tecnologías de información y comunicación (TIC's) emergentes en beneficio del usuario de la información, ya que fueron rápidamente adoptadas en la vida personal y académica de la gran mayoría de los ciudadanos; por lo que, inevitablemente hubo necesidad de expandir los alcances tradicionales de las habilidades en información a detectar, localizar, evaluar, utilizar y respetar los derechos de autor de la información recolectada tanto en fuentes impresas como digitales (Amo, Cleger y Mendoza, 2015). De esta forma, se dice que un estudiante posee MTHI cuando es capaz de involucrar las habilidades para colaborar, participar, producir y compartir información a través de las tecnologías que han evolucionado en esta era digital, entre ellas: redes sociales, blogs, microblogs, wikis, dispositivos móviles y sus diversas aplicaciones, tecnología de la nube, comunidades virtuales de aprendizaje o cursos masivos abiertos en línea (MOOCS) (Mackey y Jacobson, 2011 y 2014).

Este involucramiento tecnológico en el rastreo académico de información implica el trabajo de estar pendiente de las nuevas propuestas que emergen en la actualidad y aquellas que se proyectan para un futuro inmediato. Lo que exige, a su vez, que el estudiante desarrolle sus habilidades cognitivas como condición para encontrar sentido y pertinencia al uso de las estrategias para la obtención de MTHI (Montiel-Overall, 2006; Bury, Craig y Shujah, 2017). Nótese que la noción de metacognición, 28 de acuerdo con Flawell (1976), es el conocimiento y la dirección del pensamiento. 


\begin{tabular}{lll}
\hline \multicolumn{2}{c}{ Tabla 1. Contenidos del Taller de Metahabilidades en Información por estándar consolidado y } \\
por sesión
\end{tabular}

Fuente: Elaboración personal.

Esto significa que un individuo con experiencia metacognitiva es consciente de sus puntos débiles y fuertes, analiza sus ideas y las de los demás, analiza su atención hacia el estudio y ajusta sus estrategias de acuerdo con la tarea (Hepworth, 2015).

Así las cosas, la propuesta es consolidar los estándares internacionales más representativos de la actualidad en una matriz que permita desarrollar aquellos elementos que no estén incluidos en una u otra, pero enfatizando en la metacognición como la base para el fomento de la autodirección en el aprendizaje mediante un rastreo reflexivo de información. Esto último representa la principal crítica que le hace Piloiu (2016) a la propuesta original del metaliteracy de Mackey y Jacobson (2011 y 2014), al hacer notar que, no obstante, se hace énfasis en la importancia de los aspectos metacognitivos de las habilidades en información, no se describen estrategias para desarrollarlos. Por tal razón, en el presente estudio se busca una manera de hacerlo, 
además de alentar a los bibliotecarios académicos para que adapten su esquema de formación de usuarios a las características propias de su contexto (ver tabla 1).

Se debe tomar el reto de formar profesionistas capaces en el manejo de la tecnología, pero también en la formulación de soluciones a problemas informativos representados por los conceptos que el estudiante integra a su base de conocimiento por primera vez, aprovechando las tecnologías emergentes para la recuperación, compilación, producción y compartición de manera colaborativa de la información a través de fuentes de calidad (Mackey y Jacobson, 2014).

\section{Meronolocíl}

El presente estudio se llevó acabo en el Instituto Tecnológico y de Estudios Superiores de Monterrey, México, y su objetivo fue: comprender la manera en que el desarrollo de las metahabilidades en información coadyuva con la evolución conceptual en estudiantes de posgrado inmersos en un ámbito educativo virtual. El enfoque seleccionado fue mixto-exploratorio con un diseño secuencial, de acuerdo con lo señalado por Creswell y Plano (2017) y Tashakkori y Teddlie (2010), ya que los datos cuantitativos y cualitativos fueron recolectados en distintos momentos de la investigación, aunque no se supeditaron entre sí. La población estuvo delimitada por 102 estudiantes que cursaron la materia "Proyecto de investigación aplicada I", que forma parte de la maestría en educación en línea. La muestra de dicha población fue determinada por autoselección debido a que se invitó a los estudiantes a participar en el estudio por medio del correo electrónico (Giroux y Treblay, 2004; Hernández, Fernández y Baptista, 2014). El número final de participantes fue de 22 estudiantes.

Con la finalidad de colaborar con los procesos de enseñanza y de aprendizaje (Reale, 2015; Shumaker, 2012) se diseñó e implementó un taller de meta-habilidades en información (T-MTHI) como apoyo al programa de la materia. Estuvo conformado por seis sesiones (una por semana) vía Internet de dos horas de duración a través del programa Webex. Las sesiones fueron grabadas e incluidas en un sitio especial y los contenidos del T-MTHI se distribuyeron acorde con los estándares consolidados y su objetivo (ver tabla 1). En este caso, la modalidad en la cual el profesor bibliotecario participó fue como integrado (embedded librarian). Es decir, fungió como un cotutor, responsable de orientar a los estudiantes únicamente en los aspectos relacionados con el rastreo académico de información (Hoffman et al., 2017).

Fueron diseñados tres instrumentos. Para la parte cualitativa fueron, primero, un cuestionario de preguntas abiertas para identificar los conocimientos que los participantes tuvieron acerca de los conceptos básicos de la metodología de la investigación científica (MIC) antes y después de haber cursado el T-MTHI (pretest/postest). Su elaboración estuvo supervisada por el titular de la materia, utilizando como base para la elaboración de los reactivos bibliografía disponible en las colecciones digitales de la institución sede del estudio. Para la revisión y análisis del contenido se elaboraron tres niveles clasificación de las respuestas: no idónea, ordinaria y óptima. Esta acción se sustenta en lo propuesto por Beuchot (2009), buscando evitar los extremos 30 relacionados con un inalcanzable ideal de desempeño (univocidad vs equivocidad). 
El siguiente instrumento fue un cuestionario de cierre para los alumnos con diez preguntas abiertas, cuya finalidad fue conocer su opinión acerca del T-MTHI, su relación con la evolución conceptual y la importancia del rastreo informativo en la formación de los estudiantes. El número de reactivos de ambos instrumentos se ajustó a los contenidos y se elaboraron preguntas abiertas para fijar la atención de los participantes en las variables del estudio (Hernández et al., 2014). Para ambos cuestionarios se realizó un proceso de pilotaje con una muestra de diez alumnos de posgrado de la misma población de posgrado en línea, pero diferentes a la utilizada en el presente estudio, con la finalidad de ajustar la redacción de los reactivos y facilitar su comprensión (Díaz y Luna, 2014) (ver apéndices A y B).

Para la parte cuantitativa se adaptó y aplicó un cuestionario tipo Likert de 18 reactivos propuesto por Sackes et al. (2012). Estos investigadores lo elaboraron al estudiar las creencias motivacionales (motivational beliefs) y la influencia que ejercen sobre el cambio conceptual, ya que estas, mencionan, impulsan a los estudiantes a utilizar sus habilidades cognitivas y metacognitivas para aprender. Su objetivo fue apoyarlos para alcanzar cierta autosuficiencia en el desarrollo de conocimientos y ser persistentes, por ejemplo, para comprender conceptos de orden científico (Bandura, 1982). De hecho, las bases para la elaboración de la versión original de este instrumento fueron los lineamientos para el desarrollo de la autoeficacia de Bandura (2006). De esta manera surgió el "Efficacy Beliefs for Conceptual Change Learning Quiestionnaire", mismo que fue validado por sus autores con dos muestras de más de 300 alumnos de una clase de ciencias, donde, precisamente, se buscó conocer la autoeficacia que los participantes consideraban tener para modificar los conceptos que poseían acerca de la temática del curso después de haberlo concluido (ver apéndice C).

El proceso de adaptación y validación del instrumento utilizado en esta indagación incluyó su aplicación a una muestra de diez estudiantes de posgrado virtual diferentes a los incluidos en el pilotaje de los dos primeros instrumentos. Además, se realizó una validación mediante tres expertos (en educación, psicología y traducción) con la finalidad de procurar solidez, no solo del constructo, sino también de la utilización adecuada de las palabras en español y su equivalencia con el instrumento original en inglés (Díaz y Luna, 2014; Hernández et al., 2014). Para el análisis de los resultados se dividió en cinco categorías, donde cada una de ellas se le asignaron los valores siguientes: totalmente de acuerdo (5), de acuerdo (4), ni de acuerdo ni en desacuerdo (3), en desacuerdo (2), totalmente en desacuerdo (1). También se ajustó el factor que fue calificado por los participantes en el instrumento; es decir, se buscó específicamente conocer la actitud de los alumnos ante la evolución de aquellos conceptos básicos apoyados por el T-MTHI.

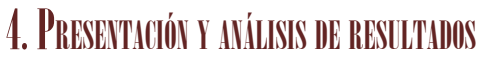

Es necesario mencionar que de los 22 estudiantes inscritos fueron 16 (11 mujeres y 5 hombres) los que concluyeron el taller, por lo que los resultados representan las respuestas de los alumnos que respondieron a todos los instrumentos como requisito de acreditación del T-MTHI. Ahora bien, con relación a los conocimientos expre- 


\begin{tabular}{|c|c|c|}
\hline Cuestionario & Pretest & Postest \\
\hline Nivel & No idóneo & Óptimo \\
\hline Respuesta & No puedo & $\begin{array}{l}\text { La variable dependiente es la que se conoce, } \\
\text { pero se desconocen los elementos que la modi- } \\
\text { fican y la variable independiente es la posible } \\
\text { respuesta a lo que modifica a la dependiente. }\end{array}$ \\
\hline Nivel & Ordinario & Ordinario \\
\hline Respuesta & $\begin{array}{l}\text { Independiente que es la variable a } \\
\text { manipular y que tendrá un efecto } \\
\text { sobre la dependiente. }\end{array}$ & $\begin{array}{l}\text { La independiente no se ve afectada; la depen- } \\
\text { diente al cambiar la independiente se produce un } \\
\text { cambio. }\end{array}$ \\
\hline Nivel & No idóneo & Ordinario \\
\hline Respuesta & Son las que forman el estudio. & $\begin{array}{l}\text { Independiente variable que no se modifica y } \\
\text { dependiente la que se verá afectada. }\end{array}$ \\
\hline
\end{tabular}

sados por los participantes acerca de los conceptos básicos de MIC, para el pretest un gran porcentaje de las respuestas relacionadas con los conceptos básicos de la materia cayeron en el nivel de "no idóneo" con un $54.3 \%$, seguido por un $44.37 \%$ de respuestas en el nivel "ordinario" y solamente un $1.32 \%$ en el "óptimo". Para el postest se constató un cambio significativo en la distribución de los porcentajes entre los tres niveles de desempeño establecidos, ya que hubo una reducción en las respuestas no idóneas de un $46.97 \%$ a $7.33 \%$, mientras que las respuestas óptimas se incrementaron de un $1.32 \%$ a $46.66 \%$, marcando un incremento del $45.34 \%$ del total de las respuestas. Finalmente, las respuestas ordinarias ocuparon el rango del $44.37 \%$, una reducción de $1.63 \%$ (ver figuras 2 y 3 ).

Cabe señalar que dentro del programa de la materia de "Proyecto de investigación aplicada I" no estuvo contemplado la revisión de ningún contenido relacionado con la MIC, por lo que los alumnos fueron alentados a revisar bibliografía por su cuenta, siguiendo las instrucciones recibidas durante el T-MTHI. De tal forma que pudo observarse cierta evolución de los conceptos expresados por los alumnos entre cada aplicación del instrumento, debido a que algunos no tenían idea acerca de la
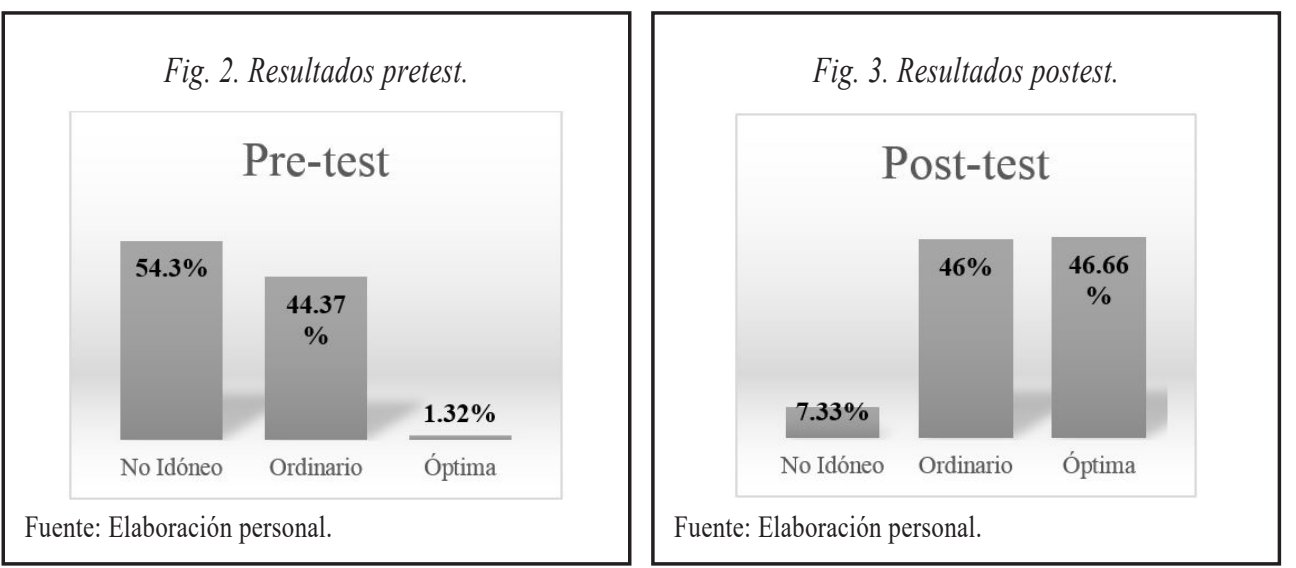
mayoría de los conceptos cuestionados y, sin embargo, al realizar el postest pudieron describirlos con más profundidad (ver tabla 2).

Para el análisis cuantitativo de los resultados del cuestionario de actitud se utilizó el programa Statistics Program for Social Sciences (SPSS), obteniendo un índice de confiabilidad de 0.830, cayendo en un nivel aceptable (Sáez, 2017). Con respecto a la validez alcanzada, dado que la participación final fue baja, no se optó por un análisis de correlación de Pearson, en parte porque no podría tener una representación significativa. Además, los mismos autores prefirieron utilizar el análisis de factores en su lugar debido a que este último no lo consideraron apropiado para analizar datos ordinales (Sackes et al., 2012). Un hallazgo interesante fue que, a pesar del pequeño número de participantes en el presente estudio, y que fue de tipo exploratorio, al analizar el factor específico para conocer la actitud de los alumnos ante la evolución de aquellos conceptos básicos apoyados por el T-MTHI, entre todos los reactivos se obtuvo un promedio de 0.788 , similar a lo obtenido por el instrumento original, pero en mediciones superiores a los 300 participantes.

En los resultados se pudo apreciar una actitud positiva hacia el papel del desarrollo de las MTHI en la evolución de los conceptos básicos revisados en el pretest y postest. En general, hubo una gran representatividad de la columna correspondiente a la opción de respuesta "totalmente de acuerdo" con un $46.44 \%$, seguido de un $41.89 \%$ de participantes que seleccionaron y estuvieron "de acuerdo". Las indecisiones fueron representadas por un $4.55 \%$, para finalmente encontrar un $1.72 \%$ de respuestas "en desacuerdo" y ninguna "totalmente en desacuerdo" (ver figura 4).

Esta actitud se vio también replicada en cada una de las dimensiones que comprenden el instrumento y en cada reactivo que las conforman (ver tabla 3).

Como se puede apreciar en la tabla 3, la dimensión que más índice de dudas marcó fue la 4: "Eficacia para la conciencia de contradicciones conceptuales", con un $12.01 \%$ de respuestas en "ni de acuerdo ni en desacuerdo" y un 4\% "en desacuerdo"; mientras que en ese mismo sentido, la dimensión 5: "Eficacia para la conciencia de la estructura conceptual", tuvo un 4.61\%; esto podría deberse al poco tiempo que se

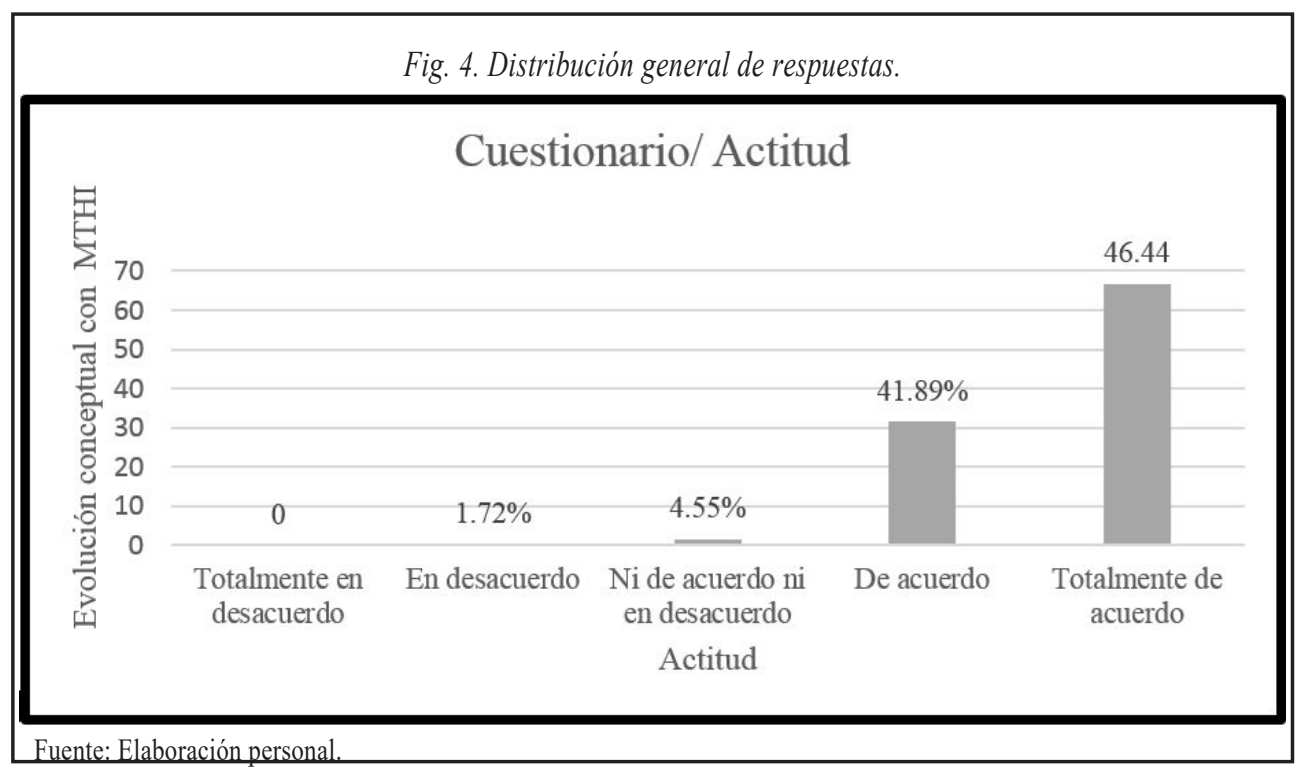




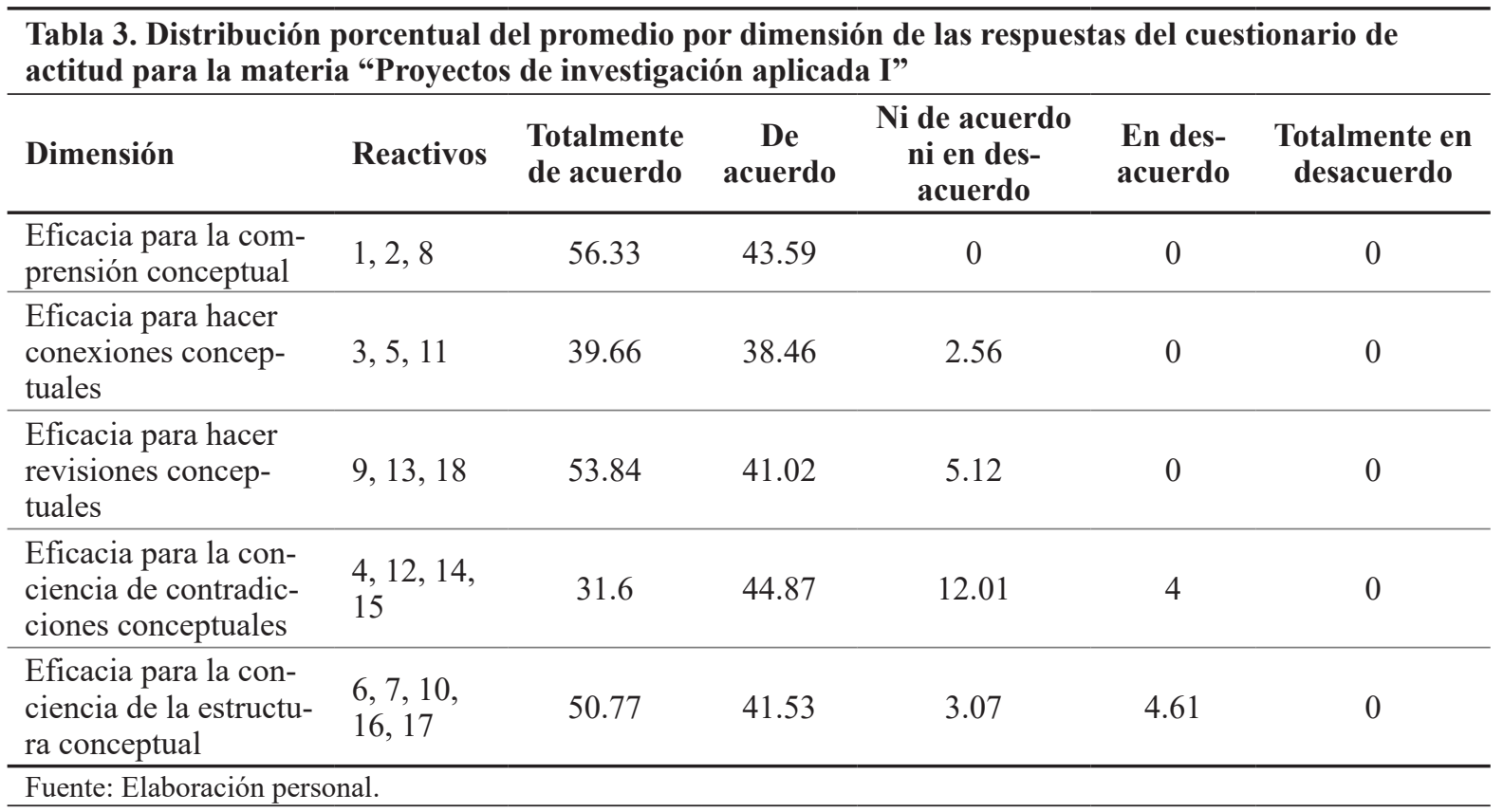

tuvo para impartir el taller (seis semanas). Por ello, para algunos participantes fue difícil apreciar posibles contradicciones entre los conceptos básicos de la MIC y los que fueron encontrando al recuperar las distintas fuentes de información.

Para el instrumento cualitativo de cierre fue posible identificar las siguientes categorías: a) conocimiento de las MTHI, en la que solamente dos alumnos reportaron tener alguna idea del significado y estándares de desempeño en las mismas; b) conocimiento de los conceptos de la MIC, donde las respuestas más frecuentes se dividieron en: 1) factores que influyen en su aprendizaje: "experiencia", "práctica" y "saber buscar información"; 2) dificultades para aprenderlos: "buscar y seleccionar fuentes adecuadas", "identificar las palabras clave", "falta de tiempo"; y, 3) alumnos con ideas preconcebidas: el $90 \%$ de los participantes indicaron que "sí, pero por falta de comprensión del tema", solamente el 10\% consideró que "no, porque no son conceptos que se enseñan con otras materias o por haber realizado alguna actividad que lo requiriera".

Para la tercera categoría, c) comportamiento informativo, indicaron: "se requiere saber rastrear información adecuadamente para comprender los conceptos que se revisan durante cualquier materia, saber buscar ahorra tiempo, utilización de un solo tipo de fuentes de información", mientras que el procedimiento de búsqueda era limitado al "catálogo de biblioteca e Internet" y sin esquema fijo. Con relación a la categoría d) apoyo de un tutor bibliotecario, las respuestas se dividieron en $91 \%$ por incluirlo en el equipo y el $9 \%$ restante se manifestó por limitarlo a ciertas materias; algunos comentarios específicos fueron: integrarlo al equipo docente, incluir la formación en MTHI al principio de la maestría, sí, pero solamente en alguna materias; $\mathrm{y}$, finalmente para la categoría e) alcances del taller de MTHI, hubo variedad en los señalamientos, tales como: disminuyó el tiempo de búsqueda, puedo identificar más rápido fuentes para el aprendizaje de conceptos, logro de autodirección y seguridad 34 en el rastreo informativo. 


\section{Clovirusulares}

Antes de iniciar con las consideraciones finales, habrá que hacer la advertencia de que las conclusiones aquí presentadas son el resultado de un estudio exploratorio, cuya finalidad fue conocer, en una primera aproximación, los efectos del desarrollo de las MTHI en la evolución conceptual en alumnos de un posgrado virtual. Entonces, los hallazgos aquí descritos solamente se aplican a los estudiantes que participaron en la misma. Sin embargo, no deja de llamar la atención que a pesar del poco tiempo que duró el experimento pudieron identificarse algunas modificaciones en la comprensión de los conceptos de la MIC e identificar una actitud positiva de los mismos participantes con relación a la percepción de sus avances en los dos instrumentos aplicados para ello. El aprendizaje es un proceso complejo, por lo que cualquier esfuerzo por apoyar su desarrollo entre los estudiantes merece la pena ser investigado, sobre todo si proviene de la bibliotecología, un área de estudio poco aprovechada para ello.

Así las cosas, una de las primeras dificultades encontradas fue la dificultad que tuvieron los alumnos, en un principio, al no saber establecer una relación entre el rastreo informativo y la elaboración de actividades académicas. Lo anterior denota que el desconocimiento de la finalidad de las MTHI en el rastreo informativo entre universitarios es un hecho recurrente. Aunque luego comprendieron las distintas posibilidades para solucionar un potencial requerimiento informativo, aprendiendo a recuperar, utilizar y organizar los datos adecuadamente (Fisher, Erdelez y McKechnie, 2009; Ford 2015). Un hecho similar fue investigado por Cid-Leal, Perpinyà y Recoder (2016) al encontrar una subutilización de fuentes y servicios de biblioteca en estudiantes de ingeniería, aunado a una deficiente habilidad para identificar información académicamente relevante, desvelando un marcado analfabetismo informativo. Lo anterior coincide con lo descrito por Luffiego et al. (1994) en su modelo de evolución conceptual, que advierte sobre la existencia de diferentes variables que pueden afectar el desarrollo de conocimientos, en la que una de esas puede ser la falta de capacidad para rastrear información (ver figura 1).

Ahora bien, el contraste de los conocimientos alcanzados durante el T-MTHI también fue señalado por Luffiego et al. (1994), en cuanto a que existen diferencias en la apreciación del fenómeno generador de conceptos, lo que hace a la capacidad personal de aprendizaje algo impredecible. Esto se pudo apreciar al encontrar un progreso notable en algunas respuestas, mientras que otras no se modificaron, 0 bien empeoraron (Perkins, 2008). De ahí que las principales dificultades percibidas por los participantes para aprender fueron la cantidad y el desconocimiento de los contenidos, confirmando que el proceso de asimilación y restructuración de nuevos conceptos es un reto, ya que el conocimiento no especializado, adquirido como producto de la primera impresión, regularmente es de tipo empírico, ordinario 0 vulgar (McPhearson et al., 2008; Ortiz, 2012). De hecho, esa fue la razón por la que se buscó triangular los resultados obtenidos por los instrumentos de recolección de datos, para conocer, desde diferentes perspectivas, lo ocurrido con el aprendizaje de los conceptos a lo largo del T-MTHI.

También se pudo encontrar una aceptación, con la presencia de un tutor bibliotecario en el equipo docente, del efecto del desarrollo de las MTHI y la evolución 
conceptual en aspectos como la aclaración, la comparación, la ampliación y la comprensión. Por otro lado, recibieron con buena disposición la organización del T-MTHI, recomendando su continuación, integración de ciertos puntos en el formato y logística de la capacitación. Esto coincide con los resultados obtenidos por Edwards y Black (2012) en un estudio dirigido a estudiantes de enfermería, donde aprobaron en general este apoyo en formato virtual, favoreciendo así el incremento en las consultas. Estos hallazgos refuerzan la importancia del papel de la metacognición como orientadora del conocimiento y el pensamiento, fortaleciendo el hecho de que un individuo con experiencia metacognitiva es consciente de sus puntos débiles y fuertes, analiza sus ideas y las de los demás, reflexiona acerca de su atención hacia el estudio y ajusta sus estrategias de acuerdo con la tarea (Barnett, McPherson y Sandieson, 2013; Flawell, 1976; Sanz de Acedo, 2011).

Aunque la educación en línea exige autodirección en el aprendizaje y en el uso de mejores fuentes, esto no significa que los estudiantes estén en condiciones de recuperar información con valor académico, sin importar su experiencia con los medios electrónicos para acceder, compilar y compartir datos (Porter, 2011; Sorgo et al., 2017). Por ello es importante diferenciar entre la habilidad para la recuperación de la información y la aptitud para manipular una computadora o dispositivos móviles, conocida como alfabetización tecnológica (Mackey y Jacobson, 2011 y 2014; Marzal y Borges, 2017; Stordy, 2015).

Y es aquí, precisamente, donde el bibliotecario académico puede apoyar en un ambiente virtual de enseñanza como un cotutor integrado (embedded librarian), dedicándose exclusivamente a fomentar entre los estudiantes el desarrollo de un proceso reflexivo de recuperación académico de la información (Waite, Gannon-Leary y Carr, 2011). Pero sin limitarse a enseñar a buscar sino a formar para saber acceder (metacognitivamente) a más y mejores fuentes de información de manera autónoma, coadyuvando con el desarrollo y la evolución de los conocimientos adquiridos (Ángel, 2016; Edwards y Black, 2012).

Dados los resultados obtenidos, surgen varias oportunidades para explorar con mayor profundidad el efecto del desarrollo de las MTHI en la evolución conceptual en un ambiente virtual de enseñanza. La primera de ellas es implementar esta capacitación y acompañamiento por un tiempo prolongado (semestres completos) y en diferentes tipos de materias. Esto proporcionará más información y permitirá verificar algunos otros aspectos de aprendizaje que pudieran ser afectados por el apoyo de un cotutor bibliotecario. Asimismo, buscar la integración formal de este tipo de actividades al programa de la materia porque, a pesar de que la asistencia no se limitó a las sesiones, que se proporcionaron distintos medios de contacto sincrónicos y asincrónicos, al no ser una actividad obligatoria, no se pudo lograr la participación de toda la población estudiantil en el T-MTHI.

Finalmente, aunque existen distintos grupos de estándares de desempeño internacionales relacionados con las habilidades en información (metahabilidades, alfabetización, etcétera), no se pretende indicar cuál es el más adecuado, ya que cada bibliotecario tiene la libertad de seleccionar el esquema de formación que mejor se adecue a sus usuarios. El único señalamiento radica alentar a seguir investigando 36 acerca del efecto del desarrollo de un proceso reflexivo de recuperación de infor- 
mación sobre la evolución de los conceptos que el alumno vaya adquiriendo en su educación y, por ende, en su aprendizaje. Esto, por supuesto, proponiendo un trabajo colaborativo con el profesorado (Hawes y Adamson, 2016).

\section{ReFreneritIs}

Álvarez, S. (2016). Martha Nussbaum y la educación en humanidades. Analecta Politica, 6(10), 167-178. Recuperado de https://dialnet.unirioja.es/descarga/articulo/5597907.pdf

Amo, J.M., Cleger, O. y Mendoza, A. (2015). Redes hipertextuales en el aula. Barcelona: Ediciones Octaedro.

Ángel, C.M. (2016). Collaboration among faculty members and community partners: Increasing the quality of online library and information science graduate programs through academic service-learning. Journal of Library y Information Services in Distance Learning, 10(1-2), 4-14. https://dx.doi.org/10.1080/1533290X.2016.1240741

Association of College and Research Libraries. (2017). Global perspectives on information literacy: Fostering a dialogue for international understanding. Chicago, IL: ACRL.

Aparicio Frutos, J.J. y Rodríguez Moneo, M. (2015). El aprendizaje humano y la memoria: una visión integrada y su correlato neurofisiológico. Espanã: Ediciones Pirámide.

Ausubel, D.P., Novak, J.D. y Hanesian, H. (1990). Psicología educativa: un punto de vista cognoscitivo. México: Trillas.

Bandura, A. (1982). Self-efficacy mechanism in human agency. American Phycologist, 37, 122147.

Bandura, A. (2006). Guide for constructing self-efficacy scales. En F. Pajares y T. Urdan (eds.), Self-efficacy of adolescents (pp. 307-337). Greenwich, CT: IAP.

Bellová, R., Melicherčíková, D. y Tomčík, P. (2017). Possible reasons for low scientific literacy of Slovak students in some natural science subjects. Research in Science y Technological Education, 36(2), 226-242. https:/dx.doi.org/10.1080/02635143.2017.1367656

Beuchot Puente, M. (2009). Hermenéutica analógica y educación multicultural. México: Plaza y Valdés.

Brodie Perry, H. (2017). Information literacy in the sciences: Faculty perception of undergraduate student skill. College y Research Libraries, 78(7), 964-977. https://dx.doi.org/10.5860/ crl.78.964

Bruce, C. (2017). Information literacy research: Dimensions of the emerging collective consciousness. Australian Academic y Research Libraries, 47(4), 220-238. https://dx.doi.org/ $10.1080 / 00048623.2016 .1253423$

Bury, S., Craig, D. y Shujah, S. (2017). Celebrating undergraduate students' research at York University: Information literacy competencies of high-achieving students. Journal of Information Literacy, 11(2), 4-27. Recuperado de https://ojs.lboro.ac.uk/JIL/article/download/ PRA-V11-I2-1/2572/

Cattaneo, K.H. (2017). Telling active learning pedagogies apart: From theory to practice. Journal of New Approaches in Educational Research, 6(2), 144-152. https://dx.doi.org/10.7821/ naer.2017.7.237

Cid-Leal, P., Perpinyà Morera, R. y Recoder Sellarès, M.J. (2016). Estrategias para combatir el analfabetismo informacional en la universidad, 3. Revista del CIDUI Congrés Internacional de Docència Universitària i Innovació, Barcelona. Recuperado de http://www.cidui.org/ revistacidui/index.php/cidui/article/view/906/868

Corsini, R. (2016). The dictionary of psychology. New York, USA: Routledge.

Creswell, J.W. y Plano Clark, V.L. (2017). Designing and conducting mixed methods research. U.S.A.: Sage. 
Delaney, G. y Bates, J. (2017). How can the university library better meet the information needs of research students? Experiences from Ulster University. New Review of Academic Librarianship, 24(1), 63-89. https://dx.doi.org/10.1080/13614533.2017.1384267

Devine, A. (2016). Literacy for visual learners: Teaching children with learning differences to read, write, communicate and create. London: Jessica Kingsley Publishers.

Díaz Barriga, A. y Luna Miranda, A.B. (2014). Metodología de la investigación educativa: aproximaciones para comprender sus estrategias. México: Ediciones Díaz de Santos.

Edwards, M.E. y Black, E.W. (2012). Contemporary instructor-librarian collaboration: A case study of an online embedded librarian implementation. Journal of Library y Information Services in Distance Learning, 6(3-4), 284-311. https://dx.doi.org/10.1080/153329 0X.2012.705690

Ferreira, T., El-Hani, C. y Silva-Filho, W. (2016). Knowledge, belief, and science education. Science y Education, 25(7/8), 775-794. https://dx.doi.org/10.1007/s11191-016-9834-6

Flavell, J.H. (1976). Metacognitive aspects of problem solving. En L.B. Resnick (ed.), The nature of intelligence (pp. 231-235). Hillsdale, NJ: Lawrence Erlbaum.

Fodor, J.A. y Pylyshyn, Z.W. (2015). Minds without meanings. U.S.A: MIT Press.

Ford, N. (2015). Introduction to information behavior. Great Britain: Facet Publishing.

Fraser, S.P. (2016). Pedagogical content knowledge (PCK): Exploring its usefulness for science lecturers in higher education. Research in Science Education, 46(1), 141-161. https://dx.doi. org/10.1007/s11165-014-9459-1

Fulkerson, D., Ariew, S. y Jacobson, T. (2017). Revisiting metacognition and metaliteracy in the ACRL framework. Communications in Information Literacy, 11(1), 21-41. https://dx.doi. org/10.15760/comminfolit.2017.11.1.45

Gilbert, S. (2017). Information literacy skills in the workplace: Examining early career advertising professionals, Journal of Business y Finance Librarianship, 22(2), 111 134, https://dx.doi.org /10.1080/08963568.2016.1258938.

Giroux, S. y Treblay, G. (2004). Metodología de las ciencias humanas. México: Fondo de Cultura Económica.

Godfree, H. y Neilson, O. (2018). School libraries matter! The missing piece in the education puzzle. Access e-Journal, 1, 28-41. Recuperado de http://www.asla.org.au/site/DefaultSite/ filesystem/documents/school_libraries_matter_access.pdf

Goodiera, S., Field, C. y Goodman, S. (2018). The need for theory evaluation in global citizenship programmes: The case of the GCSA programme. Evaluation and Program Planning, 66, 7-19. https://dx.doi.org/10.1016/j.evalprogplan.2017.08.011

Hawes, S.L. y Adamson, J.M. (2016). Flipping out over Online Library Instruction: A case study in faculty-librarian collaboration. Journal of Library y Information Services in Distance Learning, 10(3-4), 254-267. https://dx.doi.org/10.1080/1533290X.2016.1219202

Hernández, R., Fernández, C. y Baptista, P. (2014). Metodología de la investigación (6a. ed.). México: McGraw-Hill.

Hepworth, M. (2015). Developing academic information literacy for undergraduates through inquiry-based learning. Innovation in Teaching and Learning in Information and Computer Sciences, 8(2), 2-13. https://dx.doi.org/10.11120/ital.2009.08020002

Hicks, S., MacDonald, S. y Martin, E. (2017). Enhancing scientific literacy by targeting specific scientific skills. Teaching Science. The Journal of the Australian Science Teachers Association, 63(3), 26-37. ERIC Number: EJ1154812.

Hoffman, N., Beatty, S., Feng, P. y Lee, J. (2017). Teaching research skills through embedded librarianship. Reference Services Review, 45(2), 211-226. https://dx.doi.org/10.1108/RSR-072016-0045

Jacobson, T.E. y Mackey, T.P. (2016). Metaliteracy in practice. E.U.A: Neal-Schuman, American Library Association.

Kuhn, T.S. (2006). La estructura de las revoluciones cientificas. México: Fondo de Cultura Económica. 
Leliwa, S., Scangarello, J. I. y Ferreyra, Y.F. (2016). Psicología y educación (3a. ed.). Córdoba, Argentina: Editorial Brujas.

Luffiego, M., Félix, M., Ramos, F. y Soto, J. (1994). Systemic model of conceptual evolution. International Journal of Science Education, 16(3), 305-313. https://dx.doi. org/10.1080/0950069940160305.

Mackey, T.P. y Jacobson, T.E. (2011). Reframing information literacy as a metaliteracy. College y Research Libraries, 72(1), 62-78. Recuperado de https://crl.acrl.org/index.php/crl/article/ view/16132/17578

Mackey, T.P. y Jacobson, T.E. (2014). Metaliteracy. E.U.A: Neal-Schuman, American Library Association.

Majetic, C. y Pellegrino, C. (2014). When science and information literacy meet: An approach to exploring the sources of science news with non-science majors. College Teaching, 62, 107112. https://dx.doi.org/10.1080/87567555.2014.916650

Margolis, E. (2016). The conceptual mind: New directions in the study of concepts. USA, Massachusetts: MIT Press.

Marín Martínez, N. (1999). Delimitando el campo de aplicación del cambio conceptual. Enseñanza de las Ciencias, 17(1), 80-92.

Marzal, M.Á. y Borges, J. (2017). Modelos evaluativos de metaliteracy y alfabetización en información como factores de excelencia académica. Revista Española de Documentación Cientifica, 40(3), 1-17. http://dx.doi.org/10.3989/redc.2017.3.1410

Mayor, J., Suengas, A. y González Marqués, J. (1993). Estrategias metacognitivas. Aprender a aprender y aprender a pensar. España: Editorial Síntesis.

McAdoo, M.L. (2010). Building bridges. Connecting faculty, students, and college library. U.S.A: American Library Association.

McPhearson, P.T., Gill, S.P.D., Pollack, R. y Sable, J.E. (2008). Increasing scientific literacy in undergraduate education: A case study from "frontiers of science" at Columbia University. En F. Darbellay, M. Cockell, J. Billotte y F. Waldvogel (eds.), A vision of transdisciplinarity: Laying foundations for a world knowledge dialogue (pp. 148-161). Lausanne, Switzerland: EPFL Press.

Montiel-Overall, P. (2006). Teacher and Teacher-Librarian Collaboration: Moving toward Integration. Teacher Librarian, 34(2), 28-33.

Moreira, M.A. y Greca, I.M. (2003). Cambio conceptual: análisis crítico y propuestas a la luz de la teoría del aprendizaje significativo. Ciência e Educação, 9(2), 301-315. Recuperado de https://www.if.ufrgs.br/ moreira/cambioconceptual.pdf

Oliva Martínez, J.M. (1999). Algunas reflexiones sobre las concepciones alternativas y el cambio conceptual. Enseñanza de las Ciencias, 17(1), 93-107.

Ortiz Uribe, F.G. (2012). Diccionario de metodología de la investigación científica (3a. ed.). México: Limusa.

Ozdem-Yilmaz, Y. y Cavas, B. (2016). Pedagogically desirable science education: Views on inquiry-based science education in turkey. Journal of Baltic Science Education, 15(4), 506522.

Özdemir, G. y Clark, D.B. (2007). An overview of conceptual change theories. Eurasia Journal of Mathematical, Science y Technology Education, 3(4), 351-361.

Perkins, D. (2008). La escuela inteligente. España: Gedisa.

Piaget, J. (2015). La equilibración de las estructuras cognitivas: problema central del desarrollo. México: Siglo XXI Editores.

Piloiu, R.G. (2016). Rethinking the concept of "information literacy": A German perspective. Journal of Information Literacy, 10(2), 78-93. https://dx.doi.org/10.11645/10.2.2126

Posner, G., Strike, K.A., Gertzog, A. y Gertzog, W. (1982). Accommodation of scientific conception: Toward a theory of conceptual change. Science Education, 66(2), 211-227.

Pozo, J.L. y Gómez Crespo, M.A. (2006). Aprender y enseñar ciencia: del conocimiento cotidiano científico. España: Morata.

Pozo, J.I. (2008). Aprendices y maestros (2a. ed.). España: Alianza Editorial. 
Porter, B. (2011). Millennial undergraduate research strategies in web and library information retrieval systems. Journal of Web Librarianship, 5, 267-285. https://dx.doi.org/10.1080/1932 2909.2011.623538

Reale, M. (2015). Becoming an Embedded Librarian: Making Connections in the Classroom. U.S.A: American Library Association.

Richardson, J.E., Bouquin, D.R., Tmanova, L. y Wright, D. (2015). Information and informatics literacies of first-year medical students. Journal of the Medical Library Association, 103(4), 198-202.

Sackes, M., Cabe Trundle, K., Tuckman, B.W. y Krissek, L.A. (2012). Development of the efficacy beliefs for conceptual change learning questionnaire. The Journal of Experimental Education, 80(4), 338-351.

Sáez López, J.M. (2017). Investigación educativa: fundamentos teóricos, procesos y elementos prácticos: enfoque práctico con ejemplos, esencial para TFG, TFM y tesis. España: UNED, Universidad Nacional de Educación a Distancia.

Shumaker, D. (2012). Embedded librarian: Innovative strategies for taking knowledge where it's needed. U.S.A: Information Today.

Sorgo, A., Bartol, T., Dolnicar, D. y Podgornik, B. (2017). Attributes of digital natives as predictors of information literacy in higher education. British Journal of Educational Technology, 48(3), 749-767. https://dx.doi.org/10.1111/bjet.12451

Strike, K.A. y Posner, G.J. (1992). A revisionist theory of conceptual change. En R. Duschl y R. Hamilton (eds.), Philosophy of science, cognitive psychology, and educational theory and practice (pp. 147-176). Albany, NY: SUNY Press.

Taber, K.S. y Akpan, B. (2017). Science education: An international course companion. Rotterdam: Sense Publishers.

Tashakkori, A. y Teddlie, C. (2010). Mixed methods in social y behavioral research. U.S.A: Sage Editions.

Waite, K., Gannon-Leary, P. y Carr, J. (2011). The role and responsibilities of an e-tutor librarian. Journal of Library y Information Services in Distance Learning, 5, 129-148. https://dx.doi. org/ 10.1080/1533290X.2011.634980

Webb, B.S. y Hayhoe, D. (2018). Assessing the influence of an educational presentation on climate change beliefs at an Evangelical Christian College. Journal of Geoscience Education, 65(3), 272-282. https://dx.doi.org/10.5408/16-220.1

Zurkowski, P. (1974). The information service environment relationships and priorities. Washington DC, E.U.A: National Commission on Libraries and Information Science. ERIC Number: ED10039.

\section{APélingés}

\section{Ipéndice 1. Cluestionaririo utiliziddo como pretest y pastest}

1. Escriba lo que comprenda por metodología de la investigación científica.

2. ¿Qué características considera que distinguen a una investigación científica?

3. ¿Mediante qué enfoques se puede aproximar a la investigación científica?

4. ¿Qué comprende por planteamiento del problema?

5. ¿Cómo se estructura una pregunta o preguntas que definen un problema de investigación?

6. ¿Qué es un objetivo general?

7. ¿Cuáles son los objetivos específicos y qué los diferencia del objetivo general?

8. ¿Cómo definiría la justificación de una investigación? 
9. ¿Qué es una hipótesis?

10. ¿Cuál es la finalidad de la elaboración de un marco teórico en una investigación?

11. ¿Qué implica una revisión de la literatura?

12. ¿Cuál es la diferencia entre un artículo de investigación y otro que no lo es?

13. ¿Cuáles son los tipos de fuentes de información?

14. ¿En qué tipo de enfoque se utilizan los diseños preexperimentales, cuasiexperimentales y experimentales?

15. Explique brevemente el significado de variable independiente y variable dependiente.

16. ¿Qué es un instrumento de recolección de datos?

17. ¿Qué importancia tiene en una investigación definir la población y la muestra?

18. ¿En qué enfoque se utilizan las entrevistas, focus group y la observación?

19. ¿Cómo podría definir un método mixto de investigación?

20. ¿Qué información se describe en la sección del método en una investigación?

21. ¿Qué información se describe en la presentación y análisis de resultados en un estudio?

22. ¿Qué información se debe incluir en la conclusión de un estudio?

23. ¿Qué es una referencia bibliográfica?

24. Describa qué es un listado final de referencias y explique su importancia en una investigación.

\section{Ipéndice B. Cluestionaririo de cierre}

1. ¿Alguna vez había escuchado o leído al respecto de las habilidades o metahabilidades en información antes de tomar el taller? Cualquiera que sea su respuesta, por favor haga una breve descripción de lo que usted conocía o pensaba al respecto.

2. ¿Qué factores considera usted que influyen en el aprendizaje de los conceptos básicos de la metodología de la investigación científica?

3. ¿Cuáles fueron las principales dificultades a las que se enfrentó en cuanto al reconocimiento de los conceptos básicos de la metodología de la investigación científica?

4. ¿Considera que los estudiantes llegan a las aulas con ideas preconcebidas acerca de los conceptos básicos de metodología de la investigación científica?, ¿por qué?

5. ¿De qué manera afecta en la realización de sus actividades académicas el nivel de habilidad que tenga para el rastreo informativo en recursos de información digitales e impresos?

6. Antes de tomar el taller de metahabilidades en información, ¿cuál era su procedimiento para rastrear datos?

7. Tomando en cuenta la descripción de las metahabilidades en información que se hizo en las instrucciones iniciales, ¿considera que el desarrollo de las mismas podría colaborar a la modificación o evolución de los conceptos básicos que los alumnos tienen acerca de la metodología de la investigación científica?, ¿por qué?

8. ¿Consideraría adecuado que se integrara en el equipo docente de su materia a un bibliotecario como tutor para orientarlo acerca del rastreo informativo en fuentes electrónicas e impresas?, ¿por qué?

9. ¿Considera que luego de haber finalizado el taller de metahabilidades en información su procedimiento de rastreo informativo se modificó?, ¿por qué?

10. ¿Cuál es su opinión acerca del taller de metahabilidades en información que recibió a lo largo de su materia?, ¿alguna recomendación o sugerencia para mejorarlo? 


\section{Ipéndice C. Cuestionnario de actitud}

Considero que con el desarrollo de las metahabilidades en información: ${ }^{1}$

1. Yo puedo entender nuevos conceptos de la metodología de la investigación científica.

2. Yo puedo entender conceptos cada vez más complejos de la metodología de la investigación científica.

3. Soy capaz de explicar la diferencia entre los nuevos conceptos de la metodología de la investigación científica y mis creencias anteriores.

4. Yo puedo ahora reconocer si los nuevos conceptos de la metodología de la investigación científica que he aprendido tienen conflicto con mis conocimientos y creencias previas.

5. Yo puedo hacer conexiones entre los conceptos de la metodología de la investigación científica que he aprendido y los conceptos que ya conocía.

6. Yo puedo expresar cuando no comprenda los nuevos conceptos de la metodología de la investigación científica.

7. Yo puedo decir cuándo los nuevos conceptos de la metodología de la investigación científica que he aprendido me ayudan a comprender otro tipo de conceptos de este mismo tema.

8. Yo puedo continuar tratando de comprender los nuevos conceptos de la metodología de la investigación científica no obstante al principio parezcan muy difíciles de comprender.

9. Yo puedo examinar qué conocimientos de la metodología de la investigación científica ya tenía establecidos basándome en los conocimientos que he estado aprendiendo ahora.

10. Yo puedo reconocer cómo es que los conceptos de la metodología de la investigación científica que tengo actualmente influyen sobre la manera en que comprendo los nuevos conceptos de este mismo tema.

11. Yo puedo establecer si los nuevos conceptos de la metodología de la investigación científica que he aprendido concuerdan con los conceptos que ya sabía de antemano.

12. Yo puedo entender por qué algunos de los nuevos conceptos de la metodología de la investigación científica que he aprendido tienen conflicto con mis conocimientos previos acerca del tema.

13. Yo puedo alinear los conceptos de la metodología de la investigación científica que tenía hasta ahora con los nuevos conceptos que he aprendido.

14. Yo puedo percibir algún pensamiento conflictivo respecto a los conceptos de la metodología de la investigación científica.

15. Me doy cuenta cuando doy explicaciones contradictorias al describir algún concepto de la metodología de la investigación científica.

16. Yo puedo identificar la relación entre los conceptos previos sobre la metodología de la investigación científica con aquellos conceptos que he aprendido.

17. Me doy cuenta cuando los conceptos de previos sobre la metodología de la investigación científica me impiden encontrarle el sentido a los nuevos conceptos que he aprendido.

18. Yo puedo reaprender fácilmente o incluso cambiar mi forma de pensar sobre los conceptos de la metodología de la investigación científica en el contexto educativo con base en la nueva información que he adquirido. 